\title{
Stevens Johnson syndrome after carbamazepine and SJS/TEN overlap syndrome after amoxicillin: case reports and a review
}

\author{
Krystyna Romańska-Gocka ${ }^{1}$, Jacek Gocki², Waldemar Placek ${ }^{1}$, Barbara Zegarska ${ }^{3}$, Paweł Krause ${ }^{1}$
}

\begin{abstract}
${ }^{1}$ Department of Dermatology, Sexually Transmitted Diseases and Immunodermatology, Collegium Medicum in Bydgoszcz, Nicolaus Copernicus University in Toruń, Poland ${ }^{2}$ Department of Allergology, Immunology and Internal Diseases, Collegium Medicum in Bydgoszcz, Nicolaus Copernicus University in Toruń, Poland

${ }^{3}$ Department of Cosmetology, Nicolaus Copernicus University in Toruń, Poland
\end{abstract}

Submitted: 10 June 2008

Accepted: 9 September 2008

Arch Med Sci 2010; 6, 1: 130-134

DOI 10.5114/aoms.2010.13521

Copyright (c) 2010 Termedia \& Banach

\section{Abstract}

We present a case of Stevens Johnson syndrome in a child after carbamazepine application and Stevens Johnson/TEN overlap syndrome in an adult after amoxicillin application. On the basis of two reported cases we review the most commonly associated drugs, the postulated pathogenesis, clinical manifestation and management in these severe life-threatening diseases. We especially discuss the controversial systemic corticosteroid therapy. Topical care is also discussed.

Key words: Stevens Johnson syndrome.

\section{Introduction}

Stevens Johnson syndrome (SJS) and toxic epidermal necrolysis (TEN) are both acute life-threatening dermatoses characterized by extensive epidermal sloughing at the dermoepidermal junction resulting from keratinocyte apoptosis [1]. Both conditions are mostly elicited by drugs and/or their metabolites and they form part of the spectrum of erythema multiforme (EM). According to the classification proposed by Bastuji-Gari et al., cutaneous lesions of Stevens Johnson syndrome are more extensive than erythema multiforme, and epidermal necrolysis becomes a dominant feature with the involvement of up to $10 \%$ of the body surface. Severe mucous membrane lesions appear in patients with Stevens Johnson syndrome, and also in oral, conjunctival and genital areas, with a propensity for scarring and bacterial superinfection [2]. Patients with widespread macules or flat targets and 10-30\% detachment are considered to suffer from SJS/TEN overlap syndrome [3].

\section{Case reports}

\section{Case number 1 (SJS/TEN overlap syndrome)}

The 54-year-old woman was referred to our department with acute generalized skin eruptions and mucosal erosions after receiving amoxicillin for acute bronchitis. Lesions appeared rapidly and simultaneously after 2 doses of the antibiotic. Severe desquamation all over the body was observed and Nikolsky's sign was positive (Figure 1). Erythematous macular

\section{Corresponding author:}

Krystyna Romańska-Gocka, $\mathrm{MD}, \mathrm{PhD}$

Department of Dermatology, Sexually Transmitted

Diseases and Immunodermatology 5 Kurpińskiego Street 85-096 Bydgoszcz, Poland Phone: +48 525854539 Fax: +48525854018 E-mail: dermatol@poczta.onet.pl 


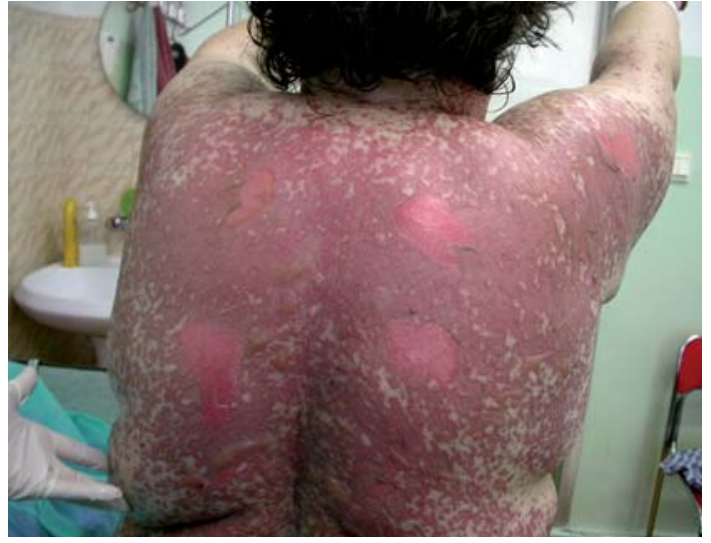

Figure 1. Positive Nikolsky’s sign

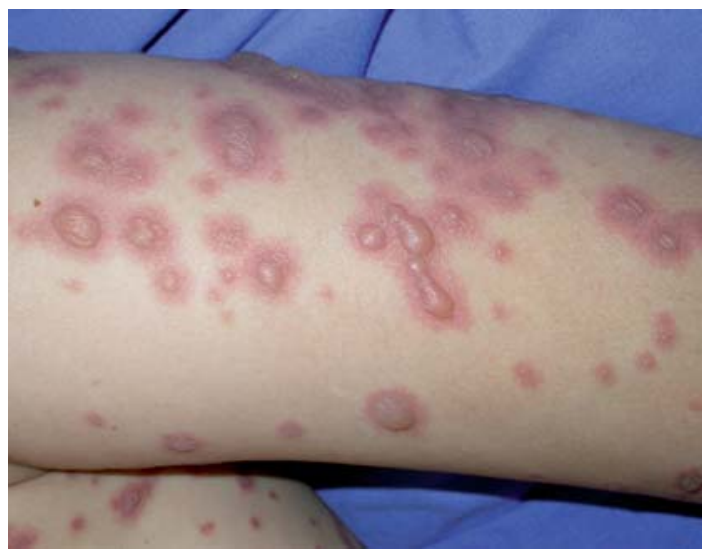

Figure 3. Erythematous macules with blisters typical for erythema multiforme/SJS

lesions showed rapid confluence. The patient showed severe mucous membrane lesions of oral, conjunctival (Figure 2) and genital areas. Eroded lesions on the lips were covered with haemorrhagic crusts. She had acute ocular findings such as purulent conjunctivitis with severe eyelid oedema and pseudomembrane formation. The general status was bad. Systemic symptoms were: fever, sore throat, and dysphagia. Our patient was also suffering from agoraphobia, insulin-dependent diabetes and arterial hypertension, making the management more difficult. The diagnosis was performed on the basis of clinical features. The main laboratory findings were: leukocytosis, sedimentation rate 64/107, the presence of bacteria, leucocytes and erythrocytes in urine analysis, significant hypoalbuminaemia and higher level of transaminases. Our patient was treated in cooperation with specialists of internal disease, an ophthalmologist and a psychiatrist. The basis of therapy was intravenous fluid replacement, steroid intravenous therapy with dexamethasone, initially $8 \mathrm{mg}$ three times a day, tapered gradually during 12 days. We also administered ciprofloxacin because of urinary tract infection. The patient required systematic control of glucose level. Topical antimicrobial dressings

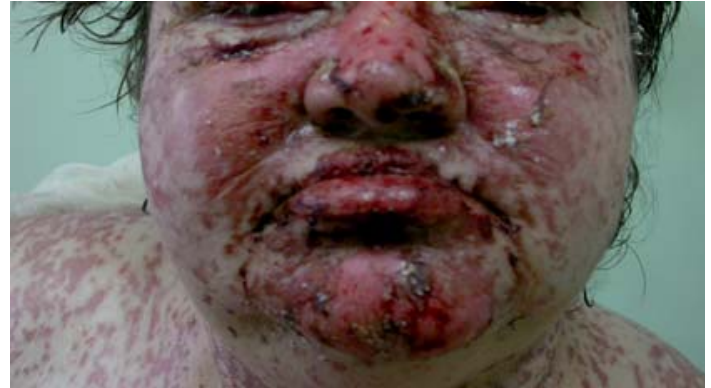

Figure 2. Mucous membrane involvement in SJS with typical erosions and haemorragic crusts on lips

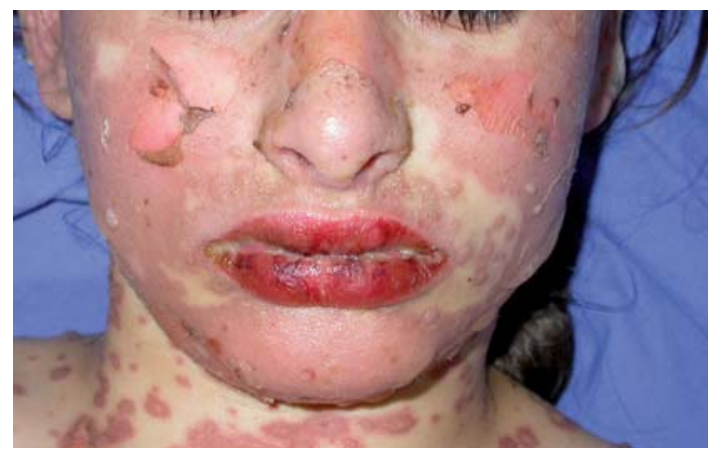

Figure 4. Mucous membrane lesions in SJS

and ophthalmological care were also very important. After therapy all symptoms and skin lesions resolved progressively. The skin lesions healed without scarring but with hyperpigmentation, during 3 weeks, and the patient left our department in a good general state.

\section{Case number 2 (Stevens Johnson syndrome)}

The 12-year-old girl was admitted to our department from the Paediatric Intensive Care Unit. She developed multiple skin lesions, but also oral mucosa involvement and severe conjunctival lesions occurred after 2 weeks of starting carbamazepine treatment for epilepsy. Her general state was severe at the beginning of treatment, with fever, headache and dysphagia. In dermatological examination, multiple erythematous lesions with blisters and large desquamation were observed (Figure 3). She had eroded lesions on the lips covered with haemorrhagic crusts and severe oedema of eyelids and lips (Figure 4). Changes in laboratory analysis included high CRP level and hypoalbuminaemia. The carbamazepine treatment was stopped immediately and the drug was replaced with Depakine Chrono. Our management was mainly supportive, with attention to fluid balance, nutritional status and pain relief. Initially, she was receiving hydrocortisone intravenously during 9 days $3 \times 150 \mathrm{mg}$ tapered systematically and later $30 \mathrm{mg}$ of prednisone tapered during 3 weeks. Other treatment included systemic antibiotic, antimicrobial dressings and 
ophthalmological treatment. All lesions healed rapidly within 2 weeks.

\section{Discussion}

Stevens Johnson syndrome (SJS) and SJS/TEN overlap syndrome are necrolytic and bullous reactions to drugs constituting part of the erythema multiforme (EM) disease spectrum. Results of a multinational study revealed that allopurinol is the drug most commonly associated with SJS or TEN [4]. Commonly associated drugs also include: antibacterial sulfonamides, anticonvulsant agents, non-steroidal anti-inflammatory drugs, aminopenicillins and quinolones [5]. This syndrome was reported less frequently after tetracyclines, methotrexate and antiretroviral drugs such as zidovudine and lamivudine [6, 7]. Among drugs recently introduced to the market, strong associations were documented for nevirapine and lamotrigine, and weaker for sertraline, pantoprazole and tramadol. The use of such drugs as first line therapies should be considered carefully, especially when safer alternative treatments exist [8].

Several mechanisms of pathogenesis are postulated. Some authors suggest that SJS and TEN are the result of deficiency concerning mechanisms involved in detoxification of reactive intermediary metabolites. Others postulate the role of several immunologically mediated mechanisms including damage mediated through CD8+ cytotoxic T cells causing epidermal necrosis [2]. The extensive keratinocyte apoptosis is at least in part mediated by the interaction of the fas receptor and fas ligand [9]. A genetic predisposition and associations with some HLA antigens have been postulated. Genetic predisposition to SJS seems to be distinct from that of TEN [10].

The diagnosis of SJS/TEN is made clinically and the first step is to withdraw the offending drugs. Clinical manifestation varies between patients, depending on the degree of skin loss and extent of mucosal involvement. As we observed, concomitant diseases such as insulin-dependent diabetes tend to worsen during SJS; they influence the general state and make treatment more difficult. If widespread cutaneous epidermal necrolysis occurs, the patient develops an imbalance of fluid and electrolyte homeostasis. Involvement of the oesophagus may result in strictures. Rarely reported symptoms are pneumothorax and mediastinal emphysema. Pneumonia is often the leading symptom in severe cases. Hepatitis and myocardial damage can be observed. Cardiac failure may result from increased cutaneous blood flow, especially in older individuals with prior cardiac disease [11].

In differential diagnosis, DRESS syndrome should be considered. Drug rash with eosinophilia and systemic symptoms (DRESS) is a multisystemic disorder resulting from reaction to certain drugs, especially anticonvulsants, but also sulfonamides, minocycline, allopurinol and others. The diagnosis of DRESS involves 3 criteria: drug-induced skin eruption, eosinophilia $>1.5 \times 10^{9} /$ l or atypical lymphocytes, and at least 1 of such systemic abnormalities as hepatitis, nephropathy, lung disease, myocardial involvement, or enlarged lymph nodes. Skin lesions could be oedematous and convert to blisters. Nikolsky's sign could be positive. The patient often has fever and malaise [12]. Maculopapular exanthema should also be considered in differential diagnosis. It is one of the most common cutaneous adverse drug reactions. One of the most frequent responsible drugs is amoxicillin, especially in patients with infectious mononucleosis. The rash usually involves the whole body but the face is often spared. These lesions do not convert to blisters and the course is mild in comparison to SJS and TEN. Some patients with an extensive maculopapular drug eruption or erythroderma may have extensive desquamation which can be confused with TEN [13]. We should think of Finkelstein's disease in infants who develop certain purpuric, oedematous targets in addition to classic target lesions. The most important differential diagnosis, especially in children and younger adults, is staphylococcal scalded skin syndrome (SSSS). Patients suffer from widespread skin erosions or display multiple vesicles. Nikolsky's sign is positive but these patients never develop mucous membrane lesions [4].

Patients suffering from SJS and TEN need careful and multidisciplinary therapeutic management. Medical therapy for SJS and TEN remains controversial, especially concerning the use of systemic corticosteroids. These drugs are the mainstay in some units but other investigators consider systemic corticosteroids to provoke prolonged wound healing and increased risk of infection, hiding early signs of sepsis, severe gastrointestinal bleeding and increased mortality $[14,15]$. Thus corticosteroids have not been recommended routinely. In contrast, a favourable influence of high doses of systemic steroids tapering over 7-10 days during the course of disease was observed by several authors [16-18]. Others only advocated their use in SJS as the skin destruction is too extensive in TEN to be reversed by the anti-inflammatory effects of steroids [19]. Nowadays, according to most authors, systemic corticosteroids are of unproven benefit in the early disease stage and are clearly deleterious in advanced forms of TEN/SJS.

Control of infection is a major task in the management of these patients. Empirical antibiotic use is generally avoided and reserved for infection confirmed by a positive culture [2]. The early use of 
broad-spectrum antibiotics immediately after admission may provoke the growth of multi-resistant microbes and the risk of Candida sepsis [19].

Cyclosporine has also been proposed as a drug with potential benefits in the management of TEN. Cyclosporine is strongly immunosuppressive and anti-apoptotic. Concerning the described cases, patients received $3 \mathrm{mg} / \mathrm{kg}$ daily for two weeks and then this dose was tapered off. Time to achieve re-epithelialization was about 12 days [20]. Cyclophosphamide is also proposed by some authors in an initial dose of $300 \mathrm{mg}$ per day [20].

Another possible method of treatment is intravenous immunoglobulin used in cases of SJS and TEN as a substance significantly decreasing mortality rates in patients. Intravenous immunoglobulin blocks fas ligand binding to the fas receptor and interferes with the downstream signalling responsible for apoptosis. In the described cases, disease progression was rapidly interrupted and all patients survived. Patients were treated with doses ranging from 0.2 to $0.75 \mathrm{~g} / \mathrm{kg}$ per day of i.v. immunoglobulin over four consecutive days [21].

Some clinicians have included daily plasmapheresis over a period of 3 days into their treatment. It is well tolerated and aims at the elimination of drugs, drug metabolites and inflammatory cytokines [19].

Topical therapy is essential to reduce loss of fluid and electrolytes and to prevent infections [21]. We use mild topical antiseptic solutions, such as chlorhexidine and silver nitrate. If biological compounds are not available, synthetic and semisynthetic bandages should be applied. It is important to keep the wounds wet to prevent progression of necrosis. Changes of dressings have to be performed under aseptic conditions once daily and a microbial test for wound infection should be performed at least three times per week from various body parts [20]. There are several dressing options available for treating TEN and SJS: porcine xenografts, synthetic dressings, dressings with occlusive silver nitrate gauze, and the traditional Vaseline impregnated gauze [2]. Topical therapy of mucous membrane includes repeated mouthwashes with antiseptic compounds and application of local anaesthetics, for example chlorhexidine rinses and white petrolatum, to lips [20].

Eye care plays a crucial role in the management of SJS/TEN patients, since ocular involvement may lead to severe complications and long-term consequences ranging from visual impairment to synechia formation and complete blindness. The most common late complication is severe dry eyes [23]. The severity of acute ocular complications does not predict late complications. The diagnosis of TEN does not imply a more severe ocular involvement or increased frequency of late ocular complications compared with SJS. Care should be taken even in mild cases. Ophthalmologists have to see the patient every day to check whether the eye is lubricated or if there are any signs of infection. Topical antibiotics should not be empirically administered unless there are clinical signs of concomitant ocular infection [23].

All patients must be daily examined for an imbalance of fluid, protein and electrolyte homeostasis. The control of red and white blood cell as well as blood coagulation factors and fibrinolytic activity is also necessary [24]. Well balanced nutrition is essential because many patients are unable to eat as a result of massive pain caused by oral mucosa lesions [20].

We conclude that early diagnosis, supportive care and careful monitoring for complications comprise crucial management in SJS.

\section{References}

1. Fromowitz JS, Ramos-Caro FA, Flowers FP. Practical guidelines for the management of toxic epidermal necrolysis and Stevens Johnson syndrome. Int I Dermatol 2007; 46: 1092-4.

2. Dalli RL, Kumar R, Kennedy P, Maitz P, Lee S, Johnson R. Toxic epidermal necrolysis/Stevens Johnson Syndrome: current trends in management. ANZ J Surg 2007; 77: 671-6.

3. Bastuji-Garin S, Rzany B, Stern RS, Shear NH, Naldi L, Roujeau JC. Clinical classification of cases of toxic epidermal necrolysis, Stevens-Johnson syndrome, and erythema multiforme. Arch Dermatol 1993; 129: 92-6.

4. Halevy S, Ghislain PD, Mockenhaupt M, et al. Allopurinol is the most common case of Stevens Johnson syndrome and toxic epidermal necrolysis in Europe and Israel. J Am Acad Dermatol 2008; 1: 25-32.

5. Roujeau JC, Kelly JP, Naldi L, et al. Medication use and the risk of Stevens Johnson syndrome or toxic epidermal necrolysis. N Engl J Med 1995; 333: 1600-7.

6. Curley RK, Verbov JL. Stevens Johnson syndrome due to tetracyclines - a case report (doxycycline) and review of the literature. Clin Exp Dermatol 1987; 12: 124-5.

7. Short RW, Agredano YZ, Choi JM, et al. Nevirapine-induced Stevens Johnson Syndrome in a pediatric patient. Pediatr Dermatol 2008; 25: 128-9.

8. Mockenhaupt M, Viboud C, Dunant A, et al. Stevens Johnson syndrome and toxic epidermal necrolysis: assessment of medication risks with emphasis on recently marketed drugs. The Euro SCAR study. J Invest Dermatol 2008; 1: 35-44.

9. Caproni M, Torchia D, Schincaglia, et al. The CD40/CD40 ligand system is expressed in the cutaneous lesions of erythema multiforme and Stevens Johnson syndrome/ toxic epidermal necrolysis spectrum. Br J Dermatol 2006; 154: 319-24.

10. Pirmohamed M, Arbuckle JB, Bowman CE, et al. Investigation into the multidimensional genetic basis of drug induced Stevens Johnson syndrome and toxic epidermal necrolysis. Pharmacogenomics 2007; 12: 1661-91.

11. Huff CL, Weston WL, Tonnesen MG. Erythema multiforme. A critical review of characteristics, diagnostic criteria and causes. J Am Acad Dermatol 1983; 8: 763-75.

12. Volpe A, Marchetta A, Cavamaschi P, et al. Hydroxychloroquine induced DRESS syndrome. Clin Rheumatol 2008; 27: 537-9. 
13. Bachot N, Roujeau JC. Differential diagnosis of severe cutaneous drug eruptions. Am J Clin Dermatol 2003; 4: 561-72.

14. Halebian P, Corder V, Madden MR, Finkelstein JL, Shires GT. Improved burn center survival of patients with toxic epidermal necrolysis managed without corticosteroids. Ann Surg 1986; 204: 503-12.

15. Cheriyan S, Patterson R, Greenberger PA, et al. The outcome of Stevens Johnson syndrome treated with corticosteroids. Allergy Proc 1995; 16: 151.

16. Crinton S, Devi K, Sridevi PK, Asokan PU. Toxic epidermal necrolysis - a retrospective study. Int J Dermatol 1997; 36: 925-32.

17. Martinez AE, Atherton DJ. High dose systemic corticosteroids can arrest recurrences of severe mucocutaneous erythema multiforme. Pediatr Dermatol 2000; 17: 87-90.

18. Patterson R, Miller M, Kaplan M, et al. Effectiveness of early therapy with corticosteroids in Stevens Johnson syndrome: experience with 41 cases and a hypothesis regarding pathogenesis. Ann Allergy 1994; 73: 27-34.

19. Rappersberger K, Foedinger D. Treatment of erythema multiforme, Stevens Johnson syndrome and toxic epidermal necrolysis. Dermatol Ther 2002; 15: 397-408.

20. Revuz J, Roujeau JC, Guilliaume JC, et al. Treatment of toxic epidermal necrolysis. Creteil's experience. Arch Dermatol 1987; 123: 1156-8.

21. Prins C, Kerdel FA, Padilla RS, et al. Treatment of toxic epidermal necrolysis with high-dose intravenous immunoglobulins: multicenter retrospective analysis of 48 consecutive cases. Arch Dermatol 2003; 139: 26-32.

22. Lin MS, Dai YS, Pwu RF, et al. Risk estimates for drugs suspected of being associated with Stevens Johnson syndrome and toxic epidermal necrolysis: a case control study. Int Med Journal 2005; 35: 188-90.

23. Yip LW, Thong BY, Lim J, et al. Ocular manifestations and complications of Stevens Johnson syndrome and toxic epidermal necrolysis: an Asian series. Allergy 2007; 62; 527-31.

24. Avakian R, Flowers FP, Araujo OE, et al. Toxic epidermal necrolysis: a review. J Am Acad Dermatol 1991; 25: 69-79. 